\title{
Electrical spin injection and detection in lateral all-semiconductor devices
}

\author{
M. Ciorga, A. Einwanger, U. Wurstbauer, D. Schuh, W. Wegscheider, and D. Weiss \\ Institute of Experimental and Applied Physics, University of Regensburg, Universitätsstrasse 31, D-93053 Regensburg, Germany
}

(Received 13 March 2009; published 29 April 2009)

\begin{abstract}
Both electrical injection and detection of spin-polarized electrons are demonstrated in a single wafer allsemiconductor GaAs-based lateral spintronic device, employing $p^{+}-(\mathrm{Ga}, \mathrm{Mn}) \mathrm{As} / n^{+}-\mathrm{GaAs}$ ferromagnetic Esaki diodes as spin aligning contacts. Spin-dependent phenomena, such as spin precession and spin-valve effect, are observed in nonlocal signal and the measurements reveal the unusual origin of the latter in the investigated devices. The conversion of spin-polarized holes into spin-polarized electrons via Esaki tunneling leaves its mark in a bias dependence of the spin-injection efficiency, which at maximum reaches the value of $50 \%$.
\end{abstract}

DOI: 10.1103/PhysRevB.79.165321

PACS number(s): 72.25.Hg, 72.25.Dc, 75.50.Pp, 85.75.-d

\section{INTRODUCTION}

The ability to inject, manipulate, and detect spin-polarized carriers by purely electrical means is at the very heart of semiconductor spintronics. ${ }^{1,2}$ Despite big progress on spin injection $^{2-11}$ into semiconductors, an all-semiconductor allelectrical injection and detection scheme has remained so far elusive. The most successful concepts for lateral spintransport devices so far employed hybrid metal/ semiconductor contacts to inject and detect spin-polarized carriers ${ }^{10,11}$ in nonmagnetic semiconducting material.

The discovery of ferromagnetism in semiconducting (Ga,Mn)As (Ref. 12) has provided the possibility to employ this material as an injector of spin-polarized carriers into a nonmagnetic semiconductor in a single all-semiconductor device. The main advantage of this approach would be the compatibility of possible future spintronic devices with the existing technology of III-V semiconductors. The biggest disadvantage of $(\mathrm{Ga}, \mathrm{Mn}) \mathrm{As}$ as a spin injector is its $p$-type character resulting in short spin-relaxation times because of the pronounced spin-orbit coupling in the valence band (VB). ${ }^{13}$ This obstacle has been recently overcome and the injection of spin-polarized electrons from (Ga,Mn)As into GaAs was detected optically using a $p^{+}-(\mathrm{Ga}, \mathrm{Mn}) \mathrm{As} / n^{+}-\mathrm{GaAs}$ Esaki diode structure as the injector contact. ${ }^{9,14,15}$ Due to the high doping on both sides of such a structure, the top of the (Ga,Mn)As VB overlaps energetically with the bottom of the GaAs conduction band (CB) and under a small reverse bias electrons from VB of (Ga,Mn)As tunnel to $\mathrm{CB}$ of GaAs (Ref. 16) leading to spin injection. In forward bias the direction of tunneling is reversed leading in turn to extraction of spins from GaAs.

In this paper we describe our experiments on lateral transport devices with Esaki diodes employed both as injecting and detecting contacts. We verify the existence of a spin transport in GaAs channel by measuring the nonlocal spinvalve (SV) effect ${ }^{10,11,17,18}$ and Hanle effect, ${ }^{10,11,18,19}$ i.e., respectively, the switching in a nonlocal signal induced by the in-plane magnetic field and the oscillation and suppression of that signal induced by a transverse magnetic field. We obtain spin polarization value of $50 \%$, which is strongly effected by an applied bias. The measurements reveal also the unusual origin of the spin-valve effect in the investigated samples that does not stem from switching between parallel and an- tiparallel configurations of magnetizations in injector and detector contacts.

The paper is organized as follows. The experimental realization of a spin-injection device and some details on performed measurements are described in Sec. II. The results of measurements are presented and discussed in Sec. III and the paper is concluded with a summary in Sec. IV.

\section{EXPERIMENTAL DETAILS}

The schematics of the sample layout as well as scanning electron microscope picture of the sample identical to the one measured are shown in Fig. 1. The device was fabricated from a single wafer grown by molecular beam epitaxy (MBE). The wafer, grown on a semi-insulating (001) GaAs substrate, consists of the following layers (in the order of growth): $500 \mathrm{~nm}$ of GaAs/AlGaAs superlattice, $250 \mathrm{~nm}$ of lightly Si-doped $n$-GaAs epilayer $\left(n=6 \times 10^{16} \mathrm{~cm}^{-3}\right), 15 \mathrm{~nm}$ of $n \rightarrow n^{+} \quad$ Si-doped GaAs transition layer $\quad\left(n^{+}=6\right.$ $\times 10^{18} \mathrm{~cm}^{-3}$ ), $8 \mathrm{~nm}$ of $n^{+}$-GaAs, $2.2 \mathrm{~nm}$ of low-temperature (LT)-grown $\mathrm{Al}_{0.36} \mathrm{Ga}_{0.64} \mathrm{As}$, and $20 \mathrm{~nm}$ of LT-grown $\mathrm{Ga}_{0.95} \mathrm{Mn}_{0.05} \mathrm{As}$. The Curie temperature of as-grown (Ga,Mn)As layer is $\sim 65 \mathrm{~K}$ as established by magnetic measurements. LT-grown (Al,Ga)As layer is used to prevent a diffusion of Mn into GaAs. The tunneling Esaki diode structure is formed by the $p$-type (Ga,Mn)As layer and $n^{+}-\mathrm{GaAs}$ layer, as shown schematically in Fig. 1(b).

The wafer has been patterned by standard photolithography and etching techniques. First a 50- $\mu \mathrm{m}$-wide mesa channel was defined along GaAs [010] direction by optical lithography and wet etched down to the semi-insulating material. Then $340 \mathrm{~nm}$ of $\mathrm{SiO}_{2}$ was deposited at $110{ }^{\circ} \mathrm{C}$ to ensure no parasitic contact between $n^{+}$and $p^{+}$layers followed by the evaporation of gold interconnecting pads. Next magnetic contacts were defined by electron-beam lithography and evaporation of $15 \mathrm{~nm}$ of $\mathrm{Ti}$ and $110 \mathrm{~nm}$ of Au. This material served as a mask during the following etching steps. In the first step the (Ga,Mn)As layer was removed by wet etching and $40 \mathrm{~nm}$ of $\mathrm{Pd}$ and $111 \mathrm{~nm}$ of Ge were evaporated for contacts to the $n$ channel. The $n^{+}$material was subsequently removed by wet chemical etching to confine the current to the lightly doped $n$-GaAs channel. The sample was then annealed for $1 \mathrm{~h}$ at $225^{\circ} \mathrm{C}$ to alloy PdGe contacts. 
(a)

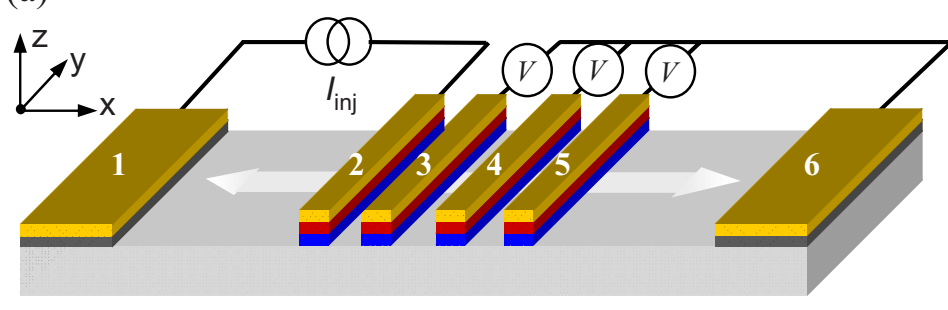

(b)

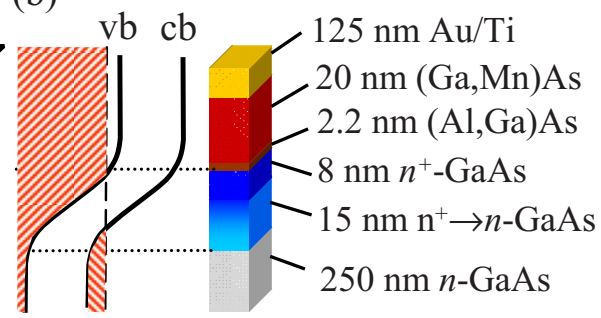

(c)
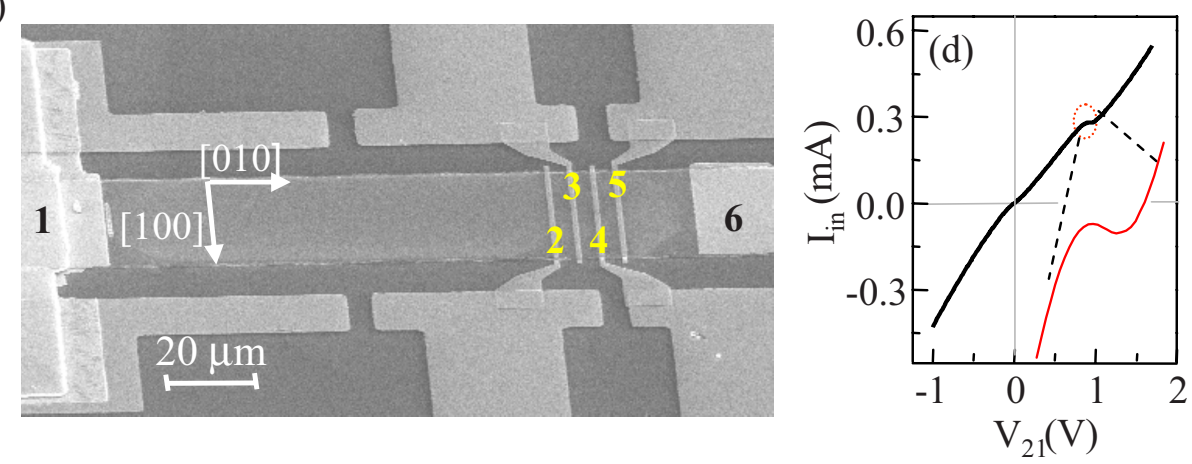

FIG. 1. (Color online) (a) A schematic of the experimental configuration (not to scale). The total of four magnetic contacts (2-5) and two nonmagnetic contacts $(1,6)$ to the $n$-GaAs channel is employed. The charge current flows only in the injector circuit whereas injected spins diffuse along $x$ in either direction of the injecting contact, as indicated by the arrows. (b) The layers forming magnetic Esaki diode contacts and the schematic of the relative position of CB and VB in the structure. Crossed areas indicate states occupied by electrons at $T=0$. The thin AlGaAs layer barrier, irrelevant to the Esaki tunneling was not included in the schematics for simplicity. (c) The scanning electron microscope picture of the tilted device identical to the one measured. The size of all magnetic contacts $(2-5)$ is $1 \times 50 \mu \mathrm{m}^{2}$ and the spacing between the centers of neighboring contacts is $5 \mu \mathrm{m}$. (d) Current-voltage characteristic measured between contacts 1 and 2 , typical also for other (Ga,Mn)As contacts. A dip in the current, characteristic for Esaki diodes, is clearly observed.

The final device consists of four magnetic (2-5) and two $(1,6)$ nonmagnetic contacts [see Figs. 1(a) and 1(c)]. Current voltage $(I-V)$ characteristics of the ferromagnetic contacts confirm the presence of Esaki tunneling [Fig. 1(d)]. Each of the magnetic contacts can be used either as spin injector or detector but for most of the measurements discussed here we use contact 2 as the injector and bias it with a constant dc $I_{21}$ flowing between this contact and contact 1 . The resulting nonlocal voltages $V_{36}, V_{46}$, and $V_{56}$ are then measured between the respective contacts. A nonlocal geometry, ${ }^{20}$ i.e., with a detector placed outside the current path, allows minimizing various spurious effects, e.g., magnetoresistance of the contacts or local Hall effect that could influence the measured signal. The dc measurements were carried out using a current source and nanovoltmeters. Depending on the type of the particular measurement the sample was mounted in a superconducting coil magnet on one of the two holders: one allowing for the in-plane and the other for the out-of-plane rotation.

For out-of-plane measurements data were taken while not sweeping through zero field. First contacts were magnetized by an in-plane field $\boldsymbol{B}_{x}=-1 \mathrm{~T}$ to ensure parallel configuration of magnetizations in injector and detector contacts. Then the field was set back to zero, the sample was rotated by $90^{\circ}$, and $\boldsymbol{B}_{z}$ was swept from zero to $-1 \mathrm{~T}$. After the sweep the sample was rotated back to the in-plane configuration and the procedure was repeated but $\boldsymbol{B}_{z}$ was now swept in the opposite direction.

\section{RESULTS AND DISCUSSION}

According to the spin-injection theory, $3,20,21$ the nonlocal voltage is a measure of a nonequilibrium spin accumulation induced in $n$-GaAs underneath the injector and diffusing in either direction of this contact, giving rise to the spinpolarized charge current in the injector circuit and pure spin current toward detector(s). At a distance $L$ from the injector it holds,

$$
V^{\mathrm{nl}}= \pm\left(P_{\mathrm{inj}} P_{\mathrm{det}} I \lambda_{\mathrm{sf}} \rho_{N} / 2 S\right) \exp \left(-L / \lambda_{\mathrm{sf}}\right),
$$

where $I$ is a bias current and $\rho_{N}, \lambda_{\mathrm{sf}}$, and $S$ are, respectively, resistivity, spin diffusion length, and the cross-section area of the nonmagnetic channel. $P_{\text {inj(det) }}$ is the spin-injection efficiency of the injector (detector) contact and expresses the polarization of the current injected at the respective contact. $+(-)$ sign corresponds to a parallel (antiparallel) configuration of magnetizations in ferromagnetic electrodes that can be switched by an in-plane magnetic field, as is done in a SV experiment.

Typical results of SV experiments on our samples are presented in Fig. 2(a). The raw data are shown, which are a sum of a spin-related signal expressed by Eq. (1) and some background signal, observed in most nonlocal SV experiments, ${ }^{10,11,17,18}$ origin of which is still not well understood. The background signal shows some slight magnetic field dependence, which however can be neglected in the shown range of the magnetic field. A clear SV-like feature is 

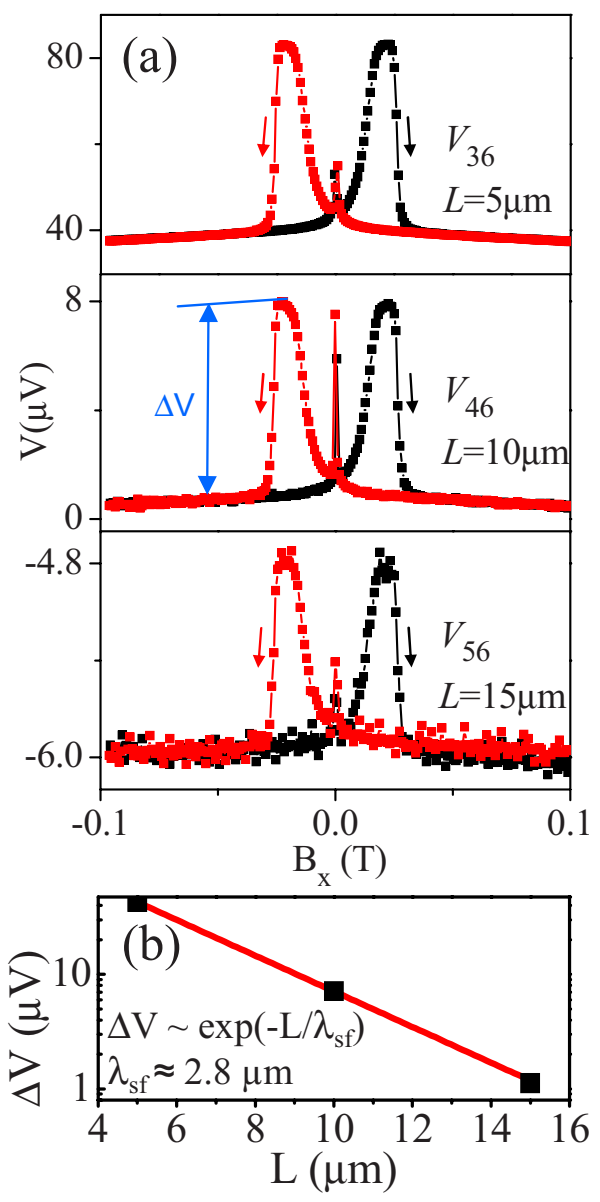

FIG. 2. (Color online) (a) Nonlocal voltage measured at three different detector contacts vs in-plane magnetic field $B_{x}$ for $I_{21}=$ $-50 \mu \mathrm{A}$. Arrows indicate the sweep directions of the respective curves. (b) Dependence of the spin-valve signal $\Delta V$ on the injectordetector separation $L$.

observed for all three detectors. The amplitude $\Delta V$ of this feature decays exponentially with the injector-detector separation in a full agreement with Eq. (1), as shown in Fig. 2(b). All curves show also a sharp feature at $0 \mathrm{~T}$. Its dependence on the $\boldsymbol{B}$-field sweeping rate suggests that it could be related to dynamic nuclear polarization (DNP) of GaAs nuclei ${ }^{22}$ due to hyperfine interaction with the spins of injected electrons ${ }^{23}$ (similar feature was also observed by Lou et al. ${ }^{10}$ ).

The most rigorous test of the system for spin transport is a demonstration of the Hanle effect, i.e., the precession and dephasing of the injected spins during transport between injector and detector in magnetic field $\boldsymbol{B}$ perpendicular to their initial orientation. ${ }^{19-21}$ The spin-related nonlocal voltage measured for parallel configuration of injector and detector can be expressed by ${ }^{19,21}$

$V_{\|}\left(x_{1}, x_{2}, B\right)=V_{0} \int_{0}^{\infty} \frac{1}{\sqrt{4 \pi D t}} e^{-\left(x_{2}-x_{1}-v_{d} t\right)^{2} / 4 D t} \cos (\Omega t) e^{-t / \tau_{s}} d t$,

where $V_{0}= \pm P_{\mathrm{inj}} P_{\mathrm{det}} I \lambda_{\mathrm{ss}} \rho_{N} / 2 S ; x_{1}$ and $x_{2}$ are, respectively, the points of injection and detection; $D$ is the spin diffusion
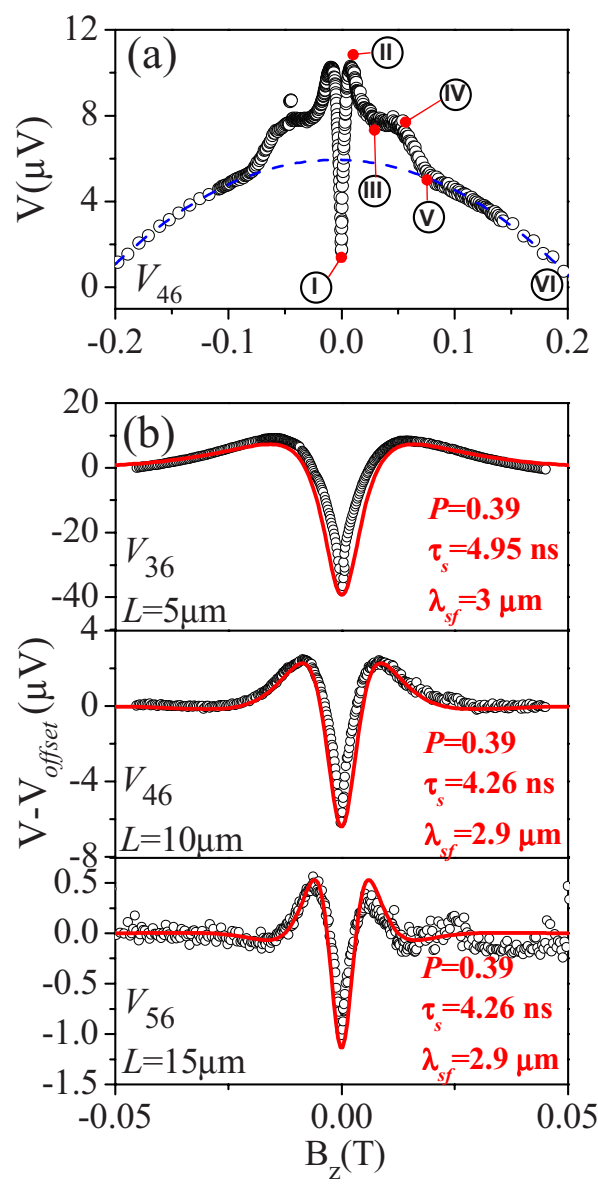

FIG. 3. (Color online) (a) The nonlocal voltage $V_{46}$ versus outof-plane magnetic field $B_{z}$. The raw data are shown. The background is fitted by a second-order polynomial (blue dashed curve). For details see text. (b) Hanle curves obtained by subtracting the offset signal from the nonlocal voltage measured at three different detectors. Solid curves are obtained from Eq. (2). with the fit parameters shown. All measurements are at $T=4.2 \mathrm{~K}$ with $I_{21}=-50 \mu \mathrm{A}$.

constant; $\tau_{s}$ is the spin relaxation time; and $\Omega=g \mu_{B} B / \hbar$ is the precession frequency.

The typical results of Hanle experiments on our samples are shown in Fig. 3. The data were combined from two separate sweeps from $\boldsymbol{B}_{z}=0$ in either direction of $\boldsymbol{B}_{z}$, as sweeping through zero resulted in very asymmetric data, showing also hysteretic behavior that could be related to DNP effects. ${ }^{22}$ Figure 3(a) shows the raw data obtained at detector 4 ( $L$ $=10 \mu \mathrm{m})$ for $I_{21}=-50 \mu \mathrm{A}$. As for the in-plane case these data are superposition of the spin signal $V_{\|}$, described by Eq. (2), and the certain offset background $V_{\text {offset }}$. At low fields strong oscillations of the spin-related signal due to Hanle effect are clearly observed. The signal decreases from its maximum value (point I), changes sign when the average spin obtains a component antiparallel to the magnetization direction of the detector (point II), and finally gets fully suppressed when all spins are dephased by $\boldsymbol{B}_{z}$ (region III-IV). At this region $V_{\|}$is then equal to zero and the measured nonlocal voltage $V=V_{\text {offset. }}$ At $B \sim 0.07 \mathrm{~T}$ magnetization of $(\mathrm{Ga}, \mathrm{Mn})$ As contacts is rotated out of the sample plane and aligned along $\boldsymbol{B}_{z}$ and the steplike feature (region IV-V) is 


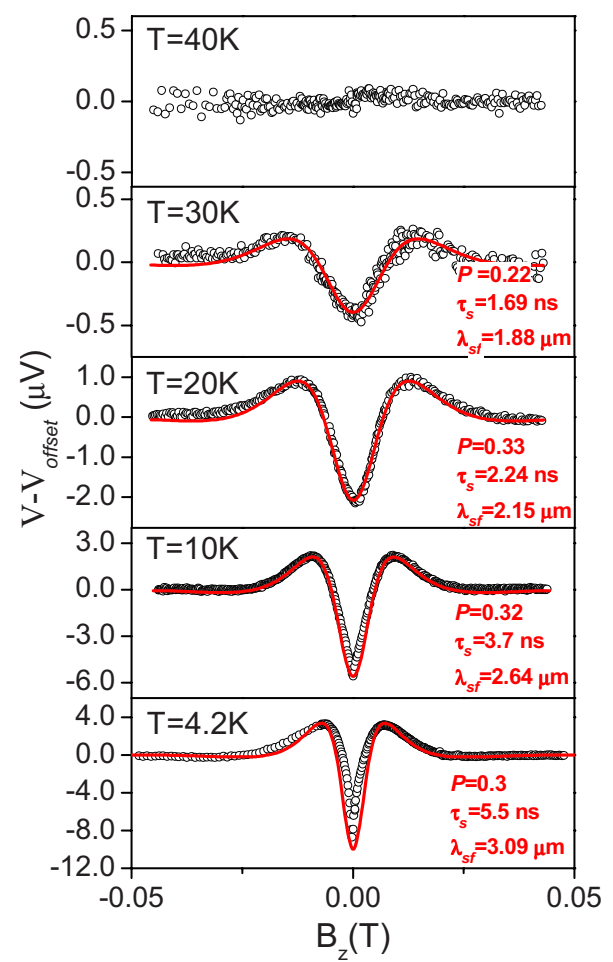

FIG. 4. (Color online) Hanle curves obtained from $V_{46}$ for $I_{21}$ $=-100 \mu \mathrm{A}$ at several different temperatures. Solid curves are obtained from Eq. (2) with the fit parameters shown.

observed in the data. At exactly this range of $\boldsymbol{B}_{z}$ a step in the resistance of the injector circuit is also observed (not shown), which can be attributed to perpendicular-to-plane tunneling anisotropic magnetoresistance (TAMR). ${ }^{24-26}$ The difference in signal between positions $\mathrm{V}$ (out-of-plane magnetization) and $I$ is then a measure of perpendicular-to-plane tunneling anisotropic spin polarization (TASP) ${ }^{2}$ At higher fields (range $\mathrm{V}-\mathrm{VI}$ ) the spin signal is saturated and measured voltage follows the background, which, similarly as in work by Lou et al. ${ }^{10}$ has a parabolic dependence on $\boldsymbol{B}_{z}$.

In Fig. 3(b) we show pure Hanle-related signal at all three detectors with the offset $V_{\text {offset }}$ removed. The signal is clearly symmetric in $\boldsymbol{B}_{z}$ suggesting that the magnetizations of injector and detector are parallel. ${ }^{19,21}$ In agreement with theory more oscillations are visible for increased injector-detector distance. Experimental data are compared with the model curves, based on Eq. (2), obtained by integrating $V_{\|}\left(x_{1}, x_{2}, B\right)$ over the width of injector and detector with $\tau_{s}, \lambda_{\text {sf }}$, and $P^{2}$ $=P_{\text {inj }} P_{\text {det }}$ being free parameters. We used the value of $g$ factor $g=-0.44$ and drift velocity $v_{d}$ was set to zero. These curves are plotted in the figure as solid red lines. From modeling we were able to estimate the spin polarization as $P$ $\sim 0.4$ and $\tau_{s}, \lambda_{\text {sf }}$ as, respectively, $\sim 4$ ns and $\sim 3 \mu \mathrm{m}$, with the latter value being consistent with SV measurements. In agreement with the model the width of the Hanle curves increases with temperature, primarily due to the decrease in $\tau_{s}$ to $1.69 \mathrm{~ns}$ at $30 \mathrm{~K}$ (Fig. 4). There is a small discrepancy between the model and the measured data near $0 \mathrm{~T}$, where the linewidth of the measured signal is smaller than that expected from the model. This also could be caused by hyperfine effects that can narrow the Hanle signal around zero

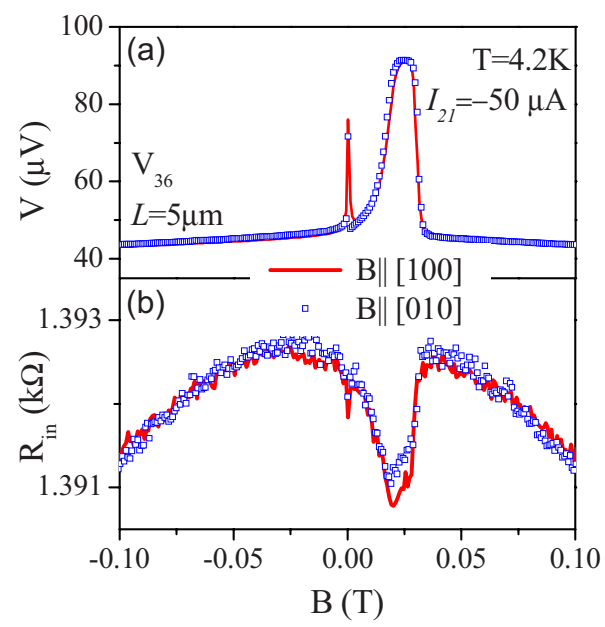

FIG. 5. (Color online) In-plane magnetic field sweeps along [100] (red line) and [010] (blue symbols) crystallographic direction. (a) Spin-valve signal at detector 3. (b) Spin-valvelike signal at injector 2 due to TAMR effect. The value of $R_{\text {in }}$ was obtained from $R_{21}$ by subtracting the resistance of the GaAs channel.

magnetic field. ${ }^{22}$ This discrepancy gets smaller when we move with the detector away from the injector, lower the bias current, or increase the temperature.

Let us now discuss the origin of the SV signal in our devices. As shape anisotropy of $(\mathrm{Ga}, \mathrm{Mn}) \mathrm{As}$ is considered to be very weak, ${ }^{27}$ it cannot be employed to switch between parallel and antiparallel configurations of magnetizations. Magnetic equivalence of [010] and [100] directions, i.e., short and long axes of our ferromagnetic contacts is clearly seen in our data. Although we could not perform standard anisotropic magnetoresistance (AMR) measurements on our samples, ${ }^{28}$ we did observe in magnetoresistance curves of the injector circuit clear spin-valvelike features due to TAMR effect so we used those data to gather some information about anisotropies in our magnetic contacts. In Fig. 5 we plot such traces and also nonlocal SV signal at detector 3, obtained from sweeps along both [010] and [100] directions. We see that the results of the sweeps are identical for both directions. What is more, the switching fields observed in TAMR-related signal are the same as those observed in nonlocal SV signal. We obtain identical results also while employing any of the remaining magnetic contacts as the injector. These results suggest that SV signal in our device is rather not a result of parallel/antiparallel switching in magnetization configuration in injector/detector contacts, as in other systems. ${ }^{10,11,17,18}$ Further information regarding the nature of the SV signal can be obtained from comparison of SV data with the results of Hanle measurements. As described in the previous paragraph from the Hanle curves we can deduce the value of the offset voltage $V_{\text {offset }}$. For any given detector contact this voltage is very close to the one measured at the top of the SV feature measured under the same experimental conditions, i.e., bias and temperature [compare, e.g., $V_{46}$ at region III-IV in Fig. 3(a) with $V_{46}$ at $\boldsymbol{B}_{x} \approx 0.02 \mathrm{~T}$ in Fig. 2(a) and see also traces in Fig. 6]. Therefore, we deduce that the spin signal at the discussed region of $\boldsymbol{B}_{x}$ is equal to zero (the $\boldsymbol{B}$ dependence of the background is negligible in the relevant 


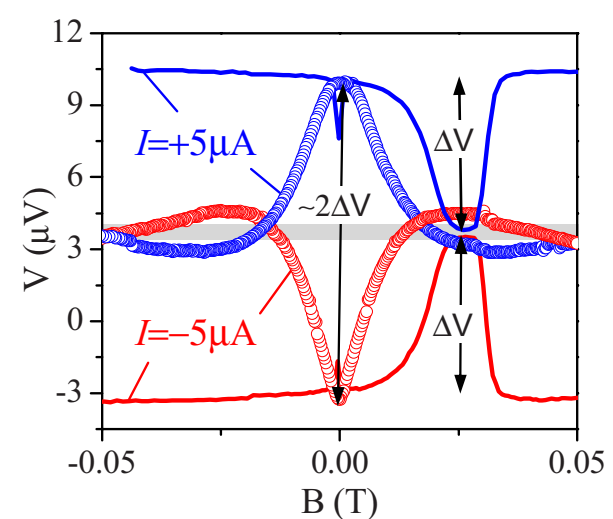

FIG. 6. (Color online) Nonlocal voltage $V_{36}$ versus in-plane field $B_{x}$ (solid lines) and out-of-plane field $B_{z}$ (symbols) for $I_{21}=$ $- \pm 5 \mu \mathrm{A}$. Gray solid bar indicates zero-spin signal.

range). This can be confirmed by comparing both Hanle and SV curves taken at opposite bias I. According to Eq. (1) reversing $I$ also reverses the sign of the measured spin signal, in similar fashion as switching magnetization configuration between parallel and antiparallel. One expects then that the signals measured for opposite $I$ will be mirror images of each other with respect to zero-spin signal. As we can see in Fig. 6 curves taken for negative bias are almost mirror images of those for positive bias with a "mirror plane" set at $V$ $\approx 3.5 \mu \mathrm{V}$, the value measured at the top (bottom for negative bias) of the SV feature, suggesting that the latter indeed originates from vanishing of the spin signal in a certain range of the magnetic field. One explanation of such behavior could be a zero net magnetization in magnetic contacts due to the formation of multidomains. Recent experiments showed evidence of a formation of multidomain structures in the magnetic reversal process in extended $(\mathrm{Ga}, \mathrm{Mn}) \mathrm{As}$ films. ${ }^{29}$ Zero-spin signals would be also observed if magnetization vectors of injector and detector were mutually orthogonal. We did try to check this by performing Hanle experiments According to the theory the orthogonal configuration should result in a Hanle signal antisymmetric in $\boldsymbol{B}_{z}$ with a zero value at $B_{z}=0 .{ }^{18,21}$ Such behavior was indeed found, appeared however to be quite volatile and, in most of our trials, on reducing $B_{x}$ to zero the signal was approaching the level observed at the parallel configuration and measured Hanle curves looked identical to the ones showed in Fig. 3. The appearance of antisymmetric Hanle signal tells us that the crossed configuration needs to be taken into account but one cannot exclude that both mentioned mechanisms, orthogonal magnetizations and multidomain formation, are in play and even may be related. The fact that all magnetic contacts show the same switching pattern in the TAMR measurements and SV signal is observed at the same field position at all three detectors suggests however that realizing the orthogonal configuration could be possible only if biasing of the one contact could change its switching behavior comparing to the others. We did observe a small influence of the bias current on switching behavior suggesting that such a scenario cannot be totally excluded but further studies, ideally involving also submicron magneto-optical measurements, would be required to fully

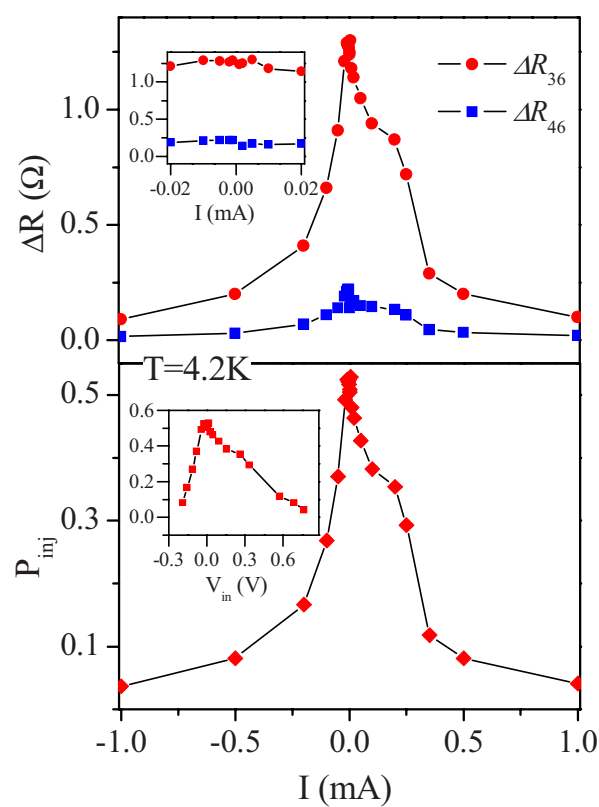

FIG. 7. (Color online) Upper panel: the value of the spin resistance $\Delta R=\Delta V / I$, measured at contacts 3 and 4 versus bias current $I_{21}$. The region of very small bias currents is shown in the inset. Lower panel: the spin-injection efficiency $P_{\text {inj }}$ (spin polarization of the injected current) versus bias current $I_{21}$. Inset: $P_{\text {inj }}$ vs bias voltage $V_{\text {in }}$ across the injecting Esaki diode. $V_{\text {in }}$ was extracted from $V_{21}$ by subtracting the voltage drop across the GaAs channel. Solid lines are only guides for the eyes.

understand the mechanism behind the switching in these small $(\mathrm{Ga}, \mathrm{Mn})$ As contacts and resulting SV effect.

As the last thing let us discuss the actual value of the spin-injection efficiency $P_{\text {inj }}$ in our devices. From model Hanle curves we extracted the value of $P$, equal to $P_{\text {inj }}$ when $P_{\text {inj }}=P_{\text {det }}$. The last equation, however, is generally not true as $P_{\text {inj }}$ is strongly bias dependent. For all used bias values $\Delta V$ $<0(\Delta V>0)$ for $I<0(I>0)$ as spin polarization is generated in GaAs by the injection (extraction) of majority spins. As expected, the absolute value of $\Delta V$ increases with increasing bias; however, the value of $\Delta R=\Delta V / I$ drops significantly at the same time, as shown in the upper panel of Fig. 7. According to Eq. (1). the latter could be attributed to the effect of the finite bias on $P_{\text {inj }}$ (we assume that $P_{\text {det }}$ is not affected). At very low bias values the dependence saturates and for the lowest measured bias of $1 \mu \mathrm{A}$ we can assume $P_{\mathrm{det}} \approx P_{\mathrm{inj}}=P$ and as a result we get $P_{\mathrm{inj}} \approx 0.5$, which is comparable to values obtained in Esaki diodes experiments with spin light-emitting diodes (LEDs) as detectors, ${ }^{30,31}$ as well as to the calculated ones ${ }^{30}$ From the bias dependence of $\Delta R$ we can then extract the bias dependence of $P_{\mathrm{inj}}$, which we plot in the lower panel of Fig. 7. Its appearance for the reverse bias is very consistent with earlier reports ${ }^{30,31}$ that explained a decrease in spin-injection efficiency by increased contribution of minority spins to the tunneling current at higher negative bias. ${ }^{30}$ At forward bias a drop in $P_{\text {inj }}$ is initially slower than for the reverse case and could be explained by inelastic tunneling processes through forbidden states in the band gap. ${ }^{16}$ The pronounced contribution of such transitions to the total current is supported by the very small peak-to valley 
ratio measured at the dip in $I-V$ curves. A dramatic drop in the $P_{\text {inj }}$ occurs at the bias of $\sim 250 \mu \mathrm{A}$. As it coincides with the dip observed at the $I-V$ characteristic of the injector, it could be explained by the fact that at this region the thermal current starts dominating over the tunneling. ${ }^{16}$

\section{SUMMARY}

In summary, we have realized the successful electrical scheme for both spin injection and detection in an allsemiconductor structure with $p^{+}-(\mathrm{Ga}, \mathrm{Mn}) \mathrm{As} / n^{+}-\mathrm{GaAs}$ ferromagnetic Esaki diodes as spin aligning contacts. From the measurements of nonlocal spin valve and spin precession we extracted the value of $\sim 50 \%$ for spin-injection efficiency for low bias currents and the value of $\sim 4.3$ ns for spin relaxation time in employed $n$-type GaAs channel. The former value was strongly decreasing with the applied bias, in a fashion expected for Esaki diode contacts. The measurements revealed also the unusual origin of the spin-valvelike effect in the spin signal that could stem from the formation of multidomain structures in the magnetic contacts during the magnetic reversal process and/or the influence of bias current on the switching process. Successful generation of a pure spin current in our type of device could offer also new possibilities in studies on spin-current-induced magnetization switching (CIMS) in (Ga,Mn)As, which attracts recently a lot of interest both because of physics involved and potential applications. ${ }^{32}$ Recent experiments on a metal system ${ }^{33}$ show that the pure spin current, having advantage of not producing any spurious charge-related effects, is as efficient in CIMS as spin-polarized charge current. As (Ga,Mn)As is generally more sensitive to CIMS than metals ${ }^{32}$ one could then envision the realization of novel all-semiconductor spintronic devices based on pure spin CIMS.

\section{ACKNOWLEDGMENTS}

We thank J. Fabian for stimulating discussions. This work was supported by the Deutscheforschungsgemeinschaft (DFG) through Sonderforschungbereich 689.
${ }^{1}$ S. Datta and B. Das, Appl. Phys. Lett. 56, 665 (1990).

${ }^{2}$ I. Žutić, J. Fabian, and S. Das Sarma, Rev. Mod. Phys. 76, 323 (2004).

${ }^{3}$ A. Fert and H. Jaffrès, Phys. Rev. B 64, 184420 (2001).

${ }^{4}$ H. J. Zhu, M. Ramsteiner, H. Kostial, M. Wassermeier, H.-P. Schönherr, and K. H. Ploog, Phys. Rev. Lett. 87, 016601 (2001).

${ }^{5}$ A. T. Hanbicki, B. T. Jonker, G. Itskos, G. Kioseoglou, and A. Petrou, Appl. Phys. Lett. 80, 1240 (2002).

${ }^{6}$ V. F. Motsnyi, W. Van Roy, G. Borghs, E. Goovaerts, and V. I. Safarov, Appl. Phys. Lett. 81, 265 (2002).

${ }^{7}$ X. Jiang, R. Wang, R. M. Shelby, R. M. Macfarlane, S. R. Bank, J. S. Harris, and S. S. P. Parkin, Phys. Rev. Lett. 94, 056601 (2005).

${ }^{8}$ R. Fiederling, M. Keim, G. Reuscher, W. Ossau, G. Schmidt, A. Waag, and L. W. Molenkamp, Nature (London) 402, 787 (1999).

${ }^{9}$ P. Van Dorpe, Z. Liu, W. Van Roy, V. F. Motsnyi, M. Sawicki, G. Borghs, and J. De Boeck, Appl. Phys. Lett. 84, 3495 (2004).

${ }^{10}$ X. Lou, C. Adelmann, S. A. Crooker, E. S. Garlid, J. Zhang, K. S. Madhukar Reddy, S. D. Flexner, C. J. Palmstrøm, and P. A. Crowell, Nat. Phys. 3, 197 (2007).

${ }^{11}$ O. M. J. van't Erve, A. T. Hanbicki, M. Holub, C. H. Li, C. Awo-Affouda, P. E. Thompson, and B. T. Jonker, Appl. Phys. Lett. 91, 212109 (2007).

${ }^{12}$ H. Ohno, A. Shen, F. Matsukura, A. Oiwa, A. Endo, S. Katsumoto, and Y. Iye, Appl. Phys. Lett. 69, 363 (1996).

${ }^{13}$ Y. Ohno, D. K. Young, B. Beschoten, F. Matsukura, H. Ohno, and D. D. Awschalom, Nature (London) 402, 790 (1999).

${ }^{14}$ M. Kohda, Y. Ohno, K. Takamura, F. Matsukura, and H. Ohno, Jpn. J. Appl. Phys., Part 2 40, L1274 (2001).

${ }^{15}$ E. Johnston-Halperin, D. Lofgreen, R. K. Kawakami, D. K. Young, L. Coldren, A. C. Gossard, and D. D. Awschalom, Phys. Rev. B 65, 041306(R) (2002).

${ }^{16}$ M. S. Sze, Physics of Semiconductor Devices (John Wiley \& Sons, New York, 1981), Chap. 9.

${ }^{17}$ F. J. Jedema, A. T. Filip, and B. J. van Wees, Nature (London)
410, 345 (2001).

${ }^{18}$ A. van Staa, J. Wulfhorst, A. Vogel, U. Merkt, and G. Meier, Phys. Rev. B 77, 214416 (2008).

${ }^{19}$ F. J. Jedema, H. B. Heersche, A. T. Filip, J. J. A. Baselmans, and B. J. van Wees, Nature (London) 416, 713 (2002).

${ }^{20}$ M. Johnson and R. H. Silsbee, Phys. Rev. Lett. 55, 1790 (1985).

${ }^{21}$ J. Fabian, A. Matos-Abiague, C. Ertler, P. Stano, and I. Zutić, Acta Phys. Slov. 57, 565 (2007).

${ }^{22}$ D. Paget and G. Lampel, G. B. Sapoval, and V. I. Safarov, Phys. Rev. B 15, 5780 (1977).

${ }^{23}$ P. Van Dorpe, W. Van Roy, J. De Boeck, and G. Borghs, Phys. Rev. B 72, 035315 (2005)

${ }^{24}$ P. Sankowski, P. Kacman, J. A. Majewski, and T. Dietl, Phys. Rev. B 75, 045306 (2007).

${ }^{25}$ C. Gould, C. Rüster, T. Jungwirth, E. Girgis, G. M. Schott, R. Giraud, K. Brunner, G. Schmidt, and L. W. Molenkamp, Phys. Rev. Lett. 93, 117203 (2004).

${ }^{26}$ M. Ciorga, M. Schlapps, A. Einwanger, S. Geißler, J. Sadowski, W. Wegscheider, and D. Weiss, New J. Phys. 9, 351 (2007).

${ }^{27}$ S. Hümpfner, K. Pappert, J. Wenisch, K. Brunner, C. Gould, G. Schmidt, L. W. Molenkamp, M. Sawicki, and T. Dietl, Appl. Phys. Lett. 90, 102102 (2007).

${ }^{28}$ The way our device is fabricated (magnetic contacts are covered with gold) does not allow to pass the current along the contact. What is more, we could not perform AMR measurements on the wafer at all as the current path through the highly doped $n^{+}$layer made interpretation of such measurements very difficult.

${ }^{29}$ D. Y. Shin, S. J. Chung, Sanghoon Lee, X. Liu, and J. K. Furdyna, Phys. Rev. Lett. 98, 047201 (2007).

${ }^{30}$ P. Van Dorpe, W. Van Roy, J. De Boeck, G. Borghs, P. Sankowski, P. Kacman, J. A. Majewski, and T. Dietl, Phys. Rev. B 72, 205322 (2005).

${ }^{31}$ M. Kohda, T. Kita, Y. Ohno, F. Matsukura, and H. Ohno, Appl. Phys. Lett. 89, 012103 (2006).

${ }^{32}$ D. Chiba, Y. Sato, T. Kita, F. Matsukura, and H. Ohno, Phys. Rev. Lett. 93, 216602 (2004).

${ }^{33}$ T. Yang, T. Kimura, and Y. Otani, Nat. Phys. 4, 851 (2008). 